\title{
Percutaneous Treatment to Inferior Vena Cava Perforation by Intracardiac Echocardiography Catheter after an Unsuccessful Conservative Approach
}

Fabricio Sarmento Vassallo ${ }^{1,2, *}$, Rodrigo França1,2, Carlos Volponi Lovatto ${ }^{2}$, Bruno Carvalho², Alessandra Cardoso ${ }^{1,2}$, Christiano Lemos da Cunha ${ }^{2}$

\section{ORCID IDs}

Vassallo FS (D) https://orcid.org/0000-0003-3915-4309
Lovatto CV (i) https://orcid.org/0000-0002-9225-2875

Lemos CC (D) https://orcid.org/0000-0001-5499-4547

\begin{abstract}
Catheter ablation is a well-established treatment for drug refractory arrhythmia, and intracardiac echocardiography is one of the tools used for this purpose. Despite a high volume of procedures performed worldwide, the incidence of vascular complications is still very low. We describe a case report of a percutaneous treatment to an inferior vena cava perforation by an intracardiac echocardiogram catheter after the failure of a conservative approach.
\end{abstract}

KEYWORDS: Catheter ablation; Atrial flutter; Vascular Surgical Procedures; Interventional ultrasonography; Inferior vena cava.

\section{INTRODUCTION}

Catheter ablation of refractory atrial tachyarrhythmias is a well-established treatment and it is performed routinely worldwide. The use of intracardiac echocardiography is already a routine support in electrophysiology laboratories during complex ablation procedures, giving direct and real time visualization of the vasculature and cardiac chambers. Despite the complexity of catheter ablation procedures, the incidence of complications is progressively being reduced, because of increasing technical expertise of the operators and the support technology.

\section{CASE REPORT}

The subject was a symptomatic 70-year-old male with previous surgical repair for an aortic aneurysm with severe mitral/aortic regurgitation. Symptoms were palpitation, shortness of breath, functional limitation, and irregular cardiac rhythm, showing mild basal pulmonary murmurs during physical exam.

1. Instituto de Cardiologia do Espírito Santo - Vitória (ES), Brazil.

2. Hospital Santa Rita - Vitória (ES), Brazil.

*Corresponding author: fabricio@arritmiaes.org

Received: Apr 21, 2021 | Accepted: May 8, 2021 
The patient described a history of recent symptoms that led him to seek for medical attention. We observed an irregular cardiac rhythm and signals of mild pulmonary congestion due to an atypical atrial flutter (AFL) at electrocardiogram.

In January 2021, we successfully performed a catheter ablation. The procedure was performed in a fasting state with general anesthesia, using the EP Tracer recording system (Cardiotek, Netherlands) supported by EnSite Velocity 5.0 (Abbott, United States) (Figs. 1 and 2) and intracardiac echocardiography (ICE) with a ViewFlex (Abbott, United States) catheter.

After successful radiofrequency ablation, all catheters were withdrawn, but a sustained hypotension, that was first interpretated as a normal reaction of general anesthesia and the tachyarrhythmia by itself, was announced by the anesthesiologist as a severe and refractory one. As we do not have any abnormal difficult during the manipulation of the catheters during the entire procedure, a complication such as a cardiac tamponade was our first diagnosis.

So, as previously performed at the beginning of the ablation, we carefully inserted the ICE probe back by the long sheath to reach the right atrium to diagnose pericardial effusion with cardiac tamponade. Since cardiac tamponade was promptly ruled out, a vascular complication was our principal hypothesis. At the larger vascular access, the $11 \mathrm{~F}$ sheath was partially pulled back outside the vein and used to make a manual nonionic contrast infusion that diagnosed an abnormal leak of this media into the retroperitoneal cavity. After this, we used a $6 \mathrm{~F}$ Pigtail catheter with a contrast-pump infusion, showing an infrarenal perforation in the inferior vena cava (IVC) (Fig. 3).

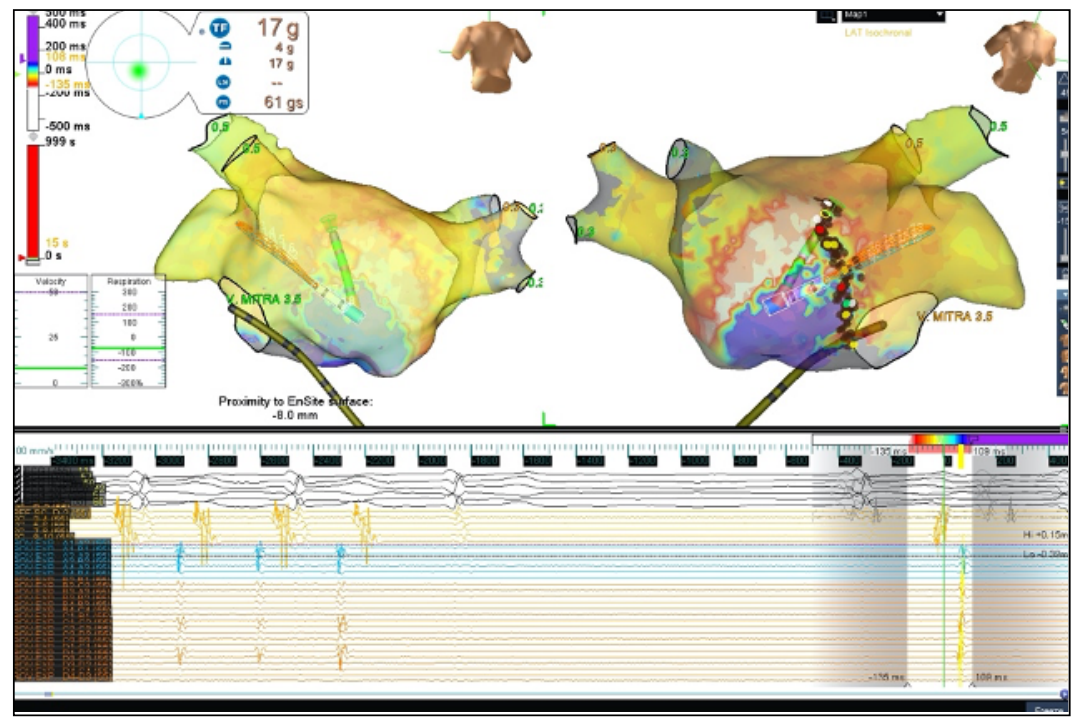

Figure 1. Isochronal tridimensional mapping of left atrial flutter in upper panel and successful catheter ablation on bottom.

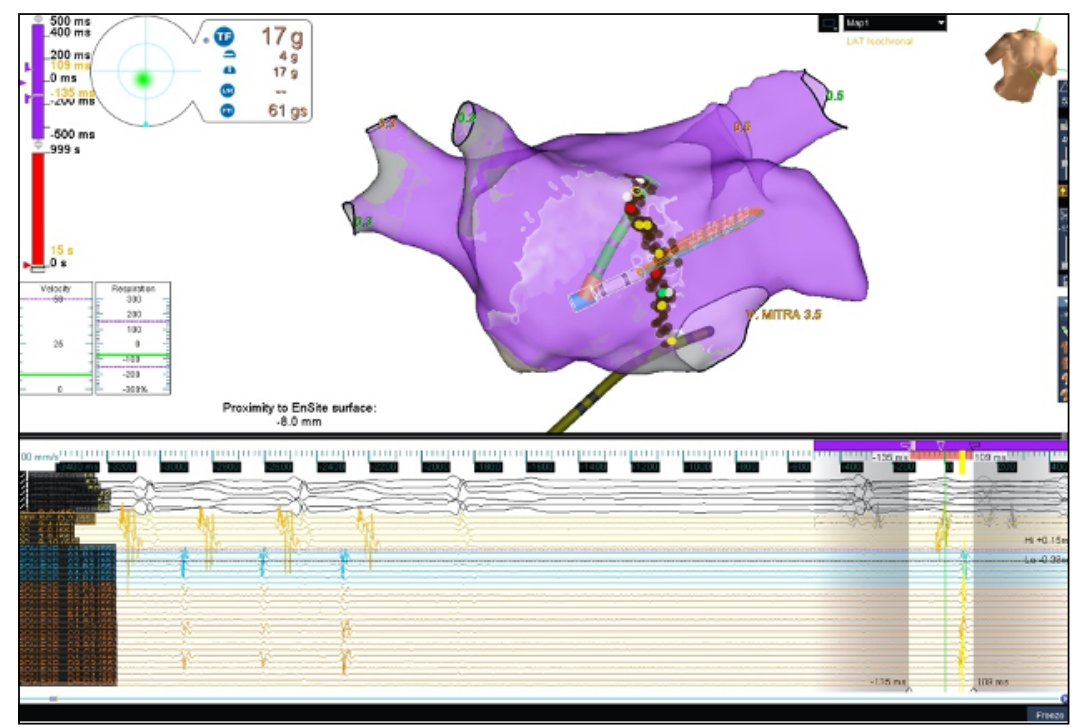

Figure 2. Propagation tridimensional mapping of left atrial flutter at upper panel and successful catheter ablation at bottom. 


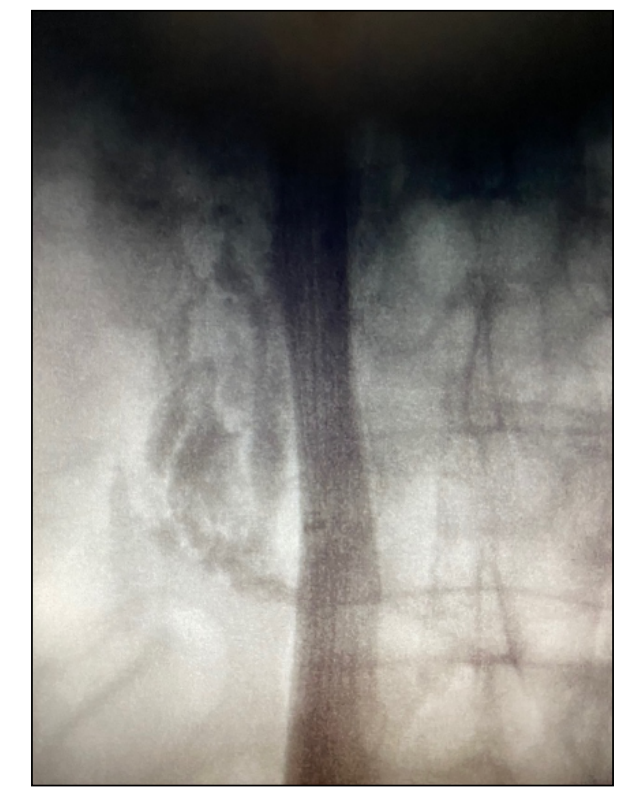

Figure 3. Infrarenal inferior vena cava perforation.

\section{MANAGEMENT}

Ephedrine, noradrenaline, and saline fluids were started to maintain adequate mean blood pressure, and antagonists of heparin and vitamin $\mathrm{K}(\mathrm{VKA})$ were administered in fresh plasma infusions and two-packed red blood cell units. A 7F short sheath was inserted at the internal right jugular vein to help in vascular management. A $14 \mathrm{~F} \mathrm{Coda}{ }^{\mathrm{TM}}$ vascular balloon of $40 \mathrm{~mm}$ in diameter and $50 \mathrm{~mm}$ in length was inserted by the left femoral vein to occlude the IVC perforation and manage the hemorrhagic shock with a conservative approach.

Several minutes after the balloon was inflated, the patient's blood pressure started to worsen. Since a conservative approach to unburden it was not possible, after measurement at the location of IVC perforation (Fig. 4), we successfully performed implantation of a Dominus $18 \mathrm{~F}$ of $22 \mathrm{~mm}$ in diameter and $50 \mathrm{~mm}$ in length Braile Biomédica ${ }^{\mathrm{TM}}$ auto-expansible grafted stent. Anesthesiologists immediately noticed a marked rise in arterial pressure due to perforation occlusion, confirmed by the injection of the contrast media (Fig. 5). Then, the stent was opened with good results, but a post-dilatation approach was necessary to totally open the stent mesh (Fig. 6).

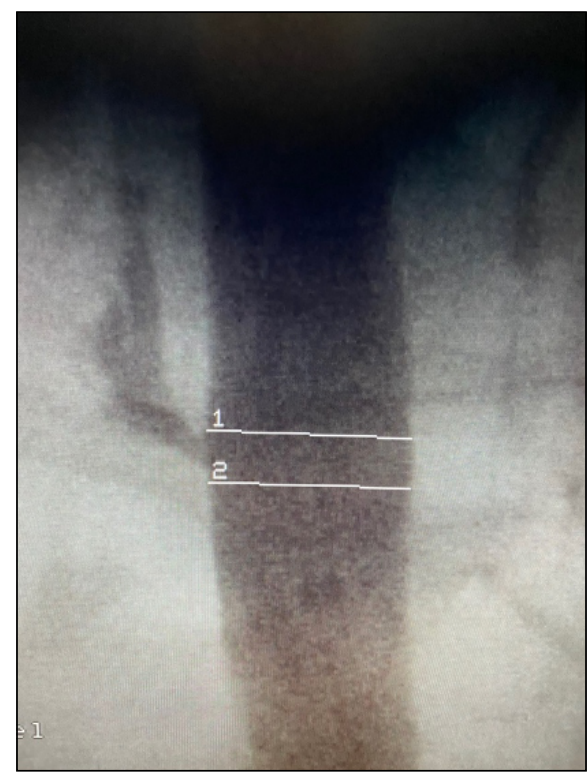

Figure 4. Inferior vena cava measurement at perforation site. Measurements at numbers 1 like $12.7 \mathrm{~cm}$ and 2 as $12.3 \mathrm{~cm}$. 


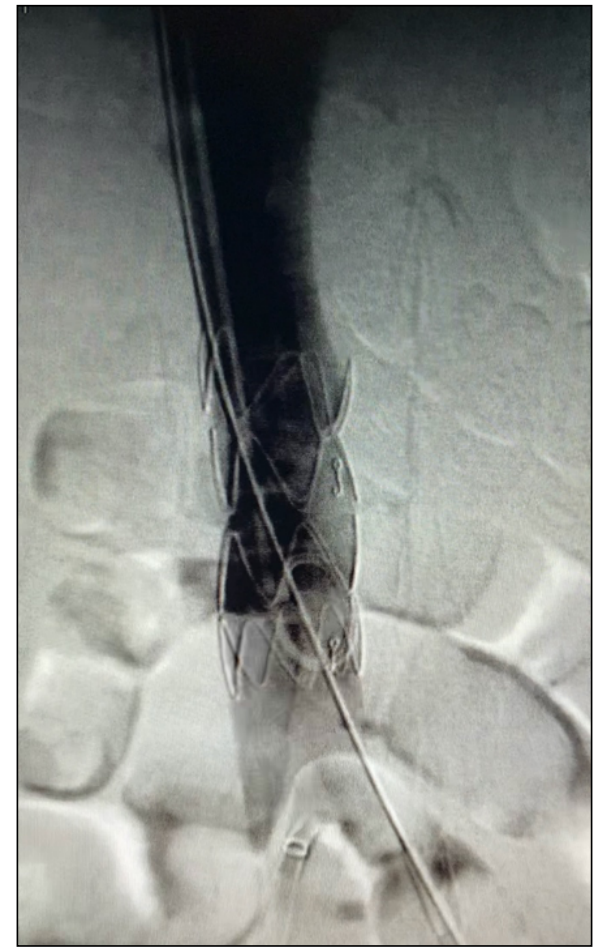

Figure 5. Digital venography showing adequate stent positioning and no contrast media leak.

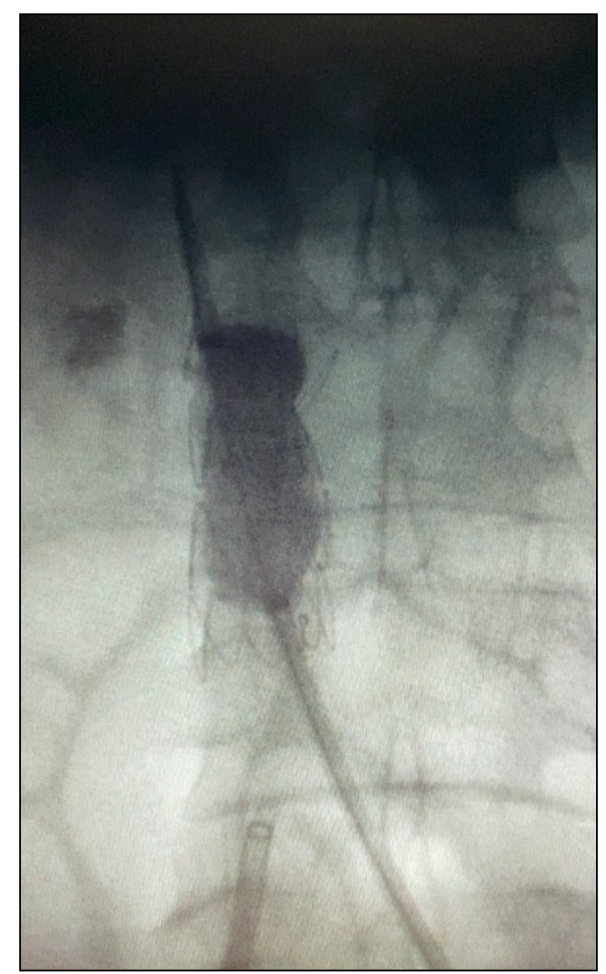

Figure 6. Post-stent implantation dilatation.

The patient was admitted to the intensive care unit (ICU) and extubated on the same day. The next day, he evaluated with abdominal distention, presenting the need for a gastric tube insertion for abdominal decompression and a 24-hour fasting state. He received a total of $210 \mathrm{~mL}$ of contrast dye media, after which urea and creatinine levels raised markedly. At the end of the second day, hemodialysis was necessary for two consecutive days, which was followed by marked improvement in renal function. A 7-day course of ceftriaxone plus metronidazole was introduced by the infectious disease department to prevent gut 
bacterial translocation, but on the first to second day after the regimen, a significant rise in C-reactive protein was observed, and a short-term 5-day course of piperacillin plus tazobactam was introduced with success. Discharge occurred at the $13^{\text {th }}$ day after the procedure, with the patient in a good general state, normal white blood cell count, mild anemia (hemoglobin $=10.4 \mathrm{~g} / \mathrm{dL}$ ), C-reactive protein $=15.3 \mathrm{mg} / \mathrm{dL}$, creatinine $=1.83 \mathrm{mg} / \mathrm{dL}$ and international normalized ratio $=2.16$.

\section{DISCUSSION}

Acute complications of catheter ablations (CA) have decreased significantly in the past few years due to improved catheter technology and increasing experience, knowledge, and expertise of the operators ${ }^{1,2}$. Nevertheless, vascular complications remain the most common adverse events related to catheter-based CA, likely due to the number and size of intravascular sheaths and the use of periprocedural anticoagulation. As an increasing movement of zero-fluoroscopy electrophysiology procedures performing worldwide ${ }^{3,4}$, the adequate use of the ICE catheter and the knowledge of possible and life-threatening complications can occur. Appropriate manipulation of the catheter and direct visualization of the venous trajectory from the sheath to the right atrial must be addressed by the operator to avoid the kind of complication described in this manuscript.

Retroperitoneal bleeding is fortunately rare, and generally managed conservatively, as most vascular complications are, but in some cases it may require administration of blood and surgical repair. Early recognition, especially if sudden hypotension develops during the procedure, is crucial, and prompt action must be taken to minimize adverse consequences. Iatrogenic injury to the vena cava is a rare but often life-threatening complication ${ }^{4-7}$. Repair often requires highly invasive surgery at the cost of high morbidity and mortality for the patient $t^{6,8,9}$.

To our knowledge, this is the second reported case of symptomatic IVC injury due to an ICE probe during CA and the first with an interventional vascular approach with a covered grafted stent with no need for open surgical repair ${ }^{3,10}$. This experience corroborates the findings of other authors that endograft stent placement in the IVC is safe, effective, and potentially lifesaving in some cases. There is a paucity of mid- and long-term data on the hemodynamic, thrombotic, and mechanical effects that arterial endograft materials may inflict on the venous system. Endovascular stent placement in the central venous system for obstruction has been well established with some predictable patency rates ${ }^{11-13}$, while there is no data from follow-up surveillance of inferior vena cava end grafts. Potential complications such as graft migration, stenosis, and thrombosis warrant close and routine surveillance ${ }^{14}$.

Before patient discharge, a control non-contrast computed tomography of the abdomen was performed, and localization of the IVC stent with a mild bilateral pleural effusion and a similar free hematic liquid in the pelvis was observed. As the patient has metallic aortic and mitral prosthetic valves, VKA in therapeutic range was the regular prescription without antiplatelets medication, and a three and a six-month doppler were assigned to monitor the stent.

\section{FOLLOW-UP}

To our knowledge, this is the first case report of an endovascular treatment for a complication due to an ICE catheter leading to an IVC perforation with acute symptomatic hemorrhagic shock. A previous related case described a more invasive approach with open abdominal surgery to repair the IVC lesion. In the present case, our patient was discharged after 14 days of hospitalization with a good clinical state and had a fast recovery. He was evaluated at seven, 14, 30, and 45 days after hospital discharge and presented a normal physical exam and normal sinus rhythm on all dates.

\section{CONCLUSION}

Catheter-induced vascular lesions during complex ablations are quite rare, and lesions to the IVC represents a daunting challenge. This case demonstrated that endovascular treatment is a good option with low morbidity. Since this complication 
is uncommon, further experience with this technique is needed to establish safety and long-term durability, and to delineate follow-up algorithms, especially as it relates to indication and duration of anticoagulation and covered graft surveillance.

\section{ACKNOWLEDGMENTS}

We thank the nurse staff Elaine Bernardes, Shirley Tessinari, Valesca Paixão, and Anderson Barcelos from the biomedical team for their efforts and dedication on this case.

\section{FUNDING}

Not applicable.

\section{DATA AVAILABILITY STATEMENT}

The datasets generated during and/or analyzed during the current study are available from the corresponding author on reasonable request.

\section{AUTHORS' CONTRIBUTION}

Critical revision: França R, Lovatto CV, Carvalho B, Cardoso A, Cunha CL; Acquisition of data: Vassallo F; Manuscript writing: Vassallo F, Fraça R; Critical revision: França R; Writing-review \& edition: Vassallo F.

\section{REFERENCES}

1. Aldhoon B, Wichterle D, Peichl P, Cihak R, Kautzner J. Complications of catheter ablation for atrial fibrillation in a high-volume center with the use of intracardiac echocardiography. Europace. 2013;15(1):24-32. https://doi.org/10.1093/europace/eus304

2. Sharma PS, Padala SK, Gunda S, Koneru JN, Ellenbogen KA. Vascular complications during catheter ablation of cardiac arrhythmias: a comparison between vascular ultrasound guided access and conventional vascular access. J Cardiovasc Electrophysiol. 2016;27(10):1160-6. https://doi.org/10.1111/jce.13042

3. Pastoricchio M, Dell'Antonio A, Zecchin M, Bianco E, Zucca A, Biloslavo A, et al. An uncommon case of inferior vena cava injury during atrial fibrillation ablation. J Surg Case Rep. 2020;2020(8):rjaa201. https://doi.org/10.1093/jscr/rjaa201

4. Di Biasi L, Horton R, Trivedi C, Mohanty P, Mohanty S, Bai R, et al. Zero Fluoroscopy Ablation of Atrial Fibrillation: A Safety and Feasibility Study. Circulation. 2014;130:A16237.

5. Saad EB, Slater C, Inácio Jr. LAO, Santos GV, Dias LC, Camanho LEC. Catheter Ablation for Treatment of Atrial Fibrillation and Supraventricular Arrhythmias Without Fluoroscopy Use: Acute Efficacy and Safety. Arq Bras Cardiol. 2020;114(6). https://doi. org/10.36660/abc.20200096

6. Wang YR, Liu YC, Chung NC, Wu S-Z, Chen M-S, Ye X-D, et al. Inferior vena cava tear during posterior spinal fusion surgery. Acta Anaesthesiol Sin. 2003;41(2):89-92.

7. Hansen CJ, Bernadas C, West MA, Ney AL, Muehlstedt S, Cohen M, et al. Abdominal vena cava injuries: outcomes remain dismal. Surgery. 2000;128(4):572-8. https://doi.org/10.1067/msy.2000.108054

8. Turpin I, State D, Schwartz A. Injuries to the inferior vena cava and their management [review]. Am J Surg. 1977;134(1):25-32. https:// doi.org/10.1016/0002-9610(77)90279-3 
9. Milikan JS, Moore EE, Cogbill TH, Kashuk JL. Inferior vena cava injuries: A continuing challenge. J Trauma. 1983;23(3):207-12. https:// doi.org/10.1097/00005373-198303000-00005

10. Kuehne J, Frankhouse J, Modrall G, Golshani S, Aziz I, Demetriades D, et al. Determinants of survival after inferior vena cava trauma. Am Surg. 1999;65(10):976-81.

11. Cheli M, Alberti D, Adriana T, Zaranko E, Colusso M, Arnoldi R, et al. Successful bleeding control by a combined conventional surgical approach and video-assisted surgery: a case report. Ann Thoracic Cardiovasc Surg. 2009;15(4):253-6.

12. Briggs CS, Morcos OC, Moriera CC, Gupta N. Endovascular treatment of iatrogenic injury to the retrohepatic inferior vena cava. Ann Vasc Surg. 2014;28(7):1794.E13-1794.E15. https://doi.org/10.1016/j.avsg.2014.04.009

13. Mansour M, Altenburg A, Haage P. Successful emergency stent implantation for superior vena cava perforation during malignant stenosis venoplasty. Cardiovasc Intervent Radiol. 2009;32(6):1312-6. https://doi.org/10.1007/s00270-009-9587-6

14. Smayra T, Otal P, Chabbert V, Chemla P, Romero M, Joffre F, et al. Long-term results of endovascular stent placement in the superior venous system. Cardiovasc Intervent Radiol. 2001;24(6):388-94. https://doi.org/10.1007/s00270-001-0055-1 